\title{
LAS VIOLENCIAS ESCOLARES EN EL ESCENARIO EDUCATIVO CHILENO. ANÁLISIS CRÍTICO DEL ESTADO DEL ARTE
}

\author{
Pablo Neut ${ }^{1}$
}

RESUMEN

La institución educativa no ha permanecido indiferente a los preceptos democratizadores imperantes en el Chile de las últimas décadas. En este contexto, la violencia escolar, al transgredir tales preceptos, representa una problemática que genera un importante interés público, mediático y académico. Sin embargo, en el tratamiento de este fenómeno ha predominado una alta dosis de confusión teórica, conceptual e, incluso, empírica. El presente trabajo tiene por objetivo analizar críticamente los estudios orientados a comprender la violencia escolar en el contexto educativo nacional. Esto se llevará a cabo a través de una revisión de las matrices teóricas preponderantes que se han ocupado de su análisis y la delimitación de múltiples lógicas o figuras de la violencia que emergen y se reproducen en las escuelas. La constatación de esta pluralidad de figuras permitirá clarificar y, al mismo tiempo, ampliar el espectro de investigaciones y líneas interpretativas abocadas al estudio de la violencia escolar en Chile.

Palabras clave: convivencia escolar, democratización, resistencia, transgresión, violencia escolar.

\section{SCHOOL VIOLENCE IN THE CHILEAN EDUCATIONAL SCENARIO. CRITICAL ANALYSIS OF THE STATE OF THE ART}

\begin{abstract}
The educational field has not remained indifferent to the democratic precepts prevailing in Chile over the last decades. In this context, the subject of school violence, upon transgressing said precepts, represents a problem that has generated significant interest among academics, the media and the general public. This topic, however, has been addressed with high levels of theoretical, conceptual and even empirical confusion. The main objective of this paper is to critically analyze studies on understanding school violence in the national educational context. This investigation conducts a critical review of theoretical matrices that have been applied to analyze this phenomenon and defines constructs of violence that emerge and are reproduced in schools. This study will be used to clarify this phenomenon, and simultaneously contribute to the field of research on the study of school violence in Chile.
\end{abstract}

Keywords: school environment, school violence, democratization, resistance, transgression.

Programa Interdisciplinario de Investigaciones en Educación (PIIE), Santiago, Chile. Contacto: pneut@piie.cl 


\section{Introducción}

La relevancia de la violencia escolar como tópico de estudio ha aumentado progresivamente en las últimas décadas, generando un acervo de conocimientos y una producción bibliográfica extensa. Sin embargo, dicho estudio se ha llevado a cabo de manera aislada y dispersa, en una sucesión ininterrumpida de publicaciones.

En el caso chileno, algunos autores han intentado sistematizar tal conocimiento indagando en la prevalencia, los tipos de violencia presentes al interior de las escuela y los factores intervinientes en las mismas (Berger, Potocnjak y Tomicic, 2011; Instituto Nacional de la Juventud, Injuv, 2017; Tijmes y Fundación Paz Ciudadana, 2012; Valdivieso, 2009). Otros han reseñado las principales investigaciones a nivel internacional que dan cuenta del fenómeno (Flores y Retamal, 2011; Zerón, 2006), así como las políticas públicas adoptadas a nivel regional para enfrentar esta problemática (Madriaza, 2006; Madriaza, Varela y Nespolo, 2011). También se han efectuado compilaciones de investigaciones acerca de la violencia escolar en los diversos países de Latinoamérica (Berger y Lisboa, 2009) y postulado nuevas perspectivas epistemológicas para su abordaje (Cofré, 2013; Flores y Retamal, 2011; Sandoval, 2006). A pesar de estos avances, se torna indispensable desentrañar las matrices teórico-conceptuales que subyacen en las interpretaciones referentes a la violencia escolar.

El presente artículo representa un esfuerzo por sistematizar el conocimiento de la violencia escolar en Chile mediante el reconocimiento de aquellas estructuras de análisis primarias que, sedimentadas disciplinariamente como paradigmas, producen esquemas de análisis generales o marcos interpretativos globales mediante los cuales se aprehende el fenómeno en estudio.

Para ello se presenta una propuesta crítica respecto de los marcos tradicionales mediante los cuales se ha trabajado el tema de la violencia escolar. Posteriormente, a partir de las críticas vertidas, se intenta responder a ellas proponiendo una delimitación entre diversas figuras de la violencia escolar. Finalmente, se presentan las investigaciones que se han abocado al análisis de cada una de 
dichas figuras en el escenario educativo chileno. No obstante el énfasis puesto en los trabajos nacionales, cuando sea necesario se referenciarán investigaciones realizadas en otras latitudes.

Mediante este recorrido se intenta clarificar y presentar un panorama general de los modos como se ha estudiado la violencia escolar en el escenario educativo nacional, analizando los límites que presentan los abordajes teóricos de los que ha sido objeto y registrando la pluralidad de figuras mediante las que se expresa la violencia en las escuelas chilenas.

\section{Análisis crítico de los marcos tradicionales de interpretación}

Las investigaciones respecto de la violencia escolar en Chile pueden ser divididas en dos categorías generales. En el primer grupo se encuentran aquellas que explican el fenómeno a partir de presupuestos estructuralistas, es decir, acudiendo a aspectos medulares del modelo de desarrollo nacional y/o dando cuenta de las mutaciones socioculturales acaecidas a nivel global. En el segundo, se inscriben aquellas que intentan comprenderlo a partir del análisis de casos particulares, identificando la prevalencia y los factores específicos que determinan su producción. Si bien cada una de estas líneas arroja luces sobre el objeto de estudio, sus enfoques presentan limitaciones que deben ser explicitadas para lograr una aprehensión cabal de la violencia escolar que se manifiesta en las escuelas chilenas.

\subsection{Interpretaciones estructurales}

Las investigaciones que han esgrimido una perspectiva estructural tienden a identificar el origen de la violencia escolar en las condiciones sociales, económicas y culturales. De acuerdo con Noel (2009), los intentos por aprehender este fenómeno han tendido a caer en dos extremos de reduccionismo interpretativo. Uno de dichos extremos estaría signado por la "metáfora de la escuela transparente". En esta versión, "la 'violencia escolar' no sería más que la irrupción, en el escenario escolar, de violencias externas y extrañas a la escuela" (p. 
39) ${ }^{2}$. La institución educativa, en esta perspectiva, recibiría el influjo directo de la violencia presente en la sociedad, sin poder resistir sus embates. En este contexto de "impotencia institucional", la violencia escolar sería un reflejo microscópico de las tensiones globales presentes en el entramado social.

Esta perspectiva estructural ha sido planteada fundamentalmente bajo tres registros de análisis o matrices de talante sociológico y/o antropológico: la teoría de la reproducción, la explicación centrada en las desigualdades estructurales y la explicación culturalista.

\subsubsection{La teoría de la reproducción y sus derivaciones}

Los análisis "reproduccionistas" son herederos de la teoría enunciada precursoramente por Bourdieu y Passeron. De acuerdo con esta, la escuela cumple la función de reproducir la estructura de clases a través de la naturalización arbitraria de la cultura de la clase dominante y su consiguiente inoculación en las clases subalternas, inoculación definida como "violencia simbólica". De esta forma, las relaciones de dominación se verían reforzadas a través de un proceso de imposición de significaciones (Bourdieu y Passeron, 1995).

Asumiendo este diagnóstico, algunos autores sostienen que las expresiones de violencia representarían la respuesta estudiantil ante la primigenia violencia simbólica perpetrada por la escuela. En el fondo, la violencia escolar representaría el intento de resistencia ejercida por los estudiantes frente a la violencia institucional que los coacciona (Ávila, 2004). Asumiendo esta perspectiva y como ejemplo paradigmático de este tipo de análisis, Llaña (2011) sentencia:

El poder materializado en los sistemas educativos como disciplinamiento tiene un oponente teórico, la resistencia (...) muchos sujetos, en situación de inferioridad, no aceptan pasivamente el control al que se sienten expuestos. Frente a un disciplinamiento, los jóvenes estudiantes, parte de una sociedad

2 El otro extremo está representado por la "metáfora de la escuela opaca", la que supone la existencia completamente autónoma de la institución escolar, en tanto microcosmos impermeable a los diversos influjos de la sociedad en la que se inserta. 
multicultural tienden a resistirlo. La resistencia, que genera prácticamente una cultura, se expresa en múltiples transgresiones que alteran el orden escolar (Llaña, 2011, pp. 64-65).

El análisis dicotómico reproducción/resistencia conlleva como corolario el diagnóstico de una realidad educativa signada por la conflictividad permanente. En efecto, si el proceso de escolarización representa la imposición de una "arbitrariedad cultural", aquellos que poseen patrones culturales distintos a los hegemónicos no padecerían pasivamente los intentos de aculturación. La tensión cultural se traduciría en una permanente transgresión de las normas, resistencias que podrían derivar en expresiones de violencia por parte de los estudiantes.

Por otra parte, la aplicación mecánica de este esquema de análisis ha redundado en la generación de un discurso reduccionista donde la dominación (violencia simbólica) y los intentos de desalienación o emancipación (resistencia) determinarían la característica esencial del proceso de escolarización ${ }^{3}$. Para los autores adscritos a esta matriz teórica, violencia simbólica y violencia escolar serían, pues, elementos constituyentes del ordenamiento escolar.

\subsubsection{Las desigualdades estructurales}

Una segunda matriz explicativa sostiene que la violencia escolar derivaría de las condiciones estructurales de desigualdad presentes en la sociedad. En este sentido, la violencia escolar sería sinónimo de violencia "general", reproducida en un escenario educativo. Característica de este tipo de análisis es la siguiente conclusión:

Desde una lectura más global del problema podríamos decir, sin embargo, que la violencia escolar es un reflejo de la 'violencia indirecta', que dimana de todo tipo de injusticias estructurales (sociales, económicas, de género, raciales...) que actúan frecuentemente como causa principal de la violencia directa. Esta

3 Sin pretender negar la presencia de esta dinámica escolar, se afirma que el reduccionismo está determinado por la negación de otras dinámicas, igualmente importantes, que se despliegan en el escenario educativo y entre los actores escolares. Para una crítica de dicho reduccionismo ver Dubet y Martuccelli (1998) y Giroux (1983). 
violencia 'indirecta, estructural o sistémica' (que es evitable) se presenta como un obstáculo invisible (Palomero y Fernández, 2001, p. 34).

La violencia escolar emanaría de condiciones desiguales preexistentes a la acción pedagógica, pero que inciden directamente en la experiencia escolar de los jóvenes, afectando particularmente a aquellos que se desarrollan en contextos de exclusión más acentuados.

Una diferencia que presenta este tipo de análisis con aquel propuesto por las teorías reproduccionistas es que, para los primeros, la escuela no cumple una función específica -la denominada "violencia simbólica"- en la perpetuación de la desigualdad que actúa como causa precursora de la violencia. En consecuencia, la institución educativa sería un ente pasivo que, en un acto reflejo, recibe las heterogéneas injusticias sociales indirectas y las traduce en violencia escolar directa.

Al mismo tiempo, subyace a este tipo de análisis la percepción de una violencia que se manifiesta de manera generalizada en el espacio escolar. La violencia escolar representaría un "campo magmático" que impregna todo el escenario educativo, sin una forma definida y cuya proveniencia posee un carácter exógeno. Bajo estos supuestos, la interpretación de las desigualdades estructurales se distancia nuevamente de la propuesta reproduccionista según la cual la violencia escolar tiene un origen o causa específica (la violencia simbólica y/o la resistencia estudiantil), unos actores determinados (las autoridades escolares y los estudiantes) y una direccionalidad particular.

\subsubsection{La cultura de la violencia y el "vacío" posmoderno}

Una tercera vertiente pretende dar cuenta de la violencia escolar a través de las mutaciones culturales acaecidas a nivel global en las últimas décadas.

Desde una perspectiva que adquiere un cariz conservador y un tono de nostálgica denuncia se ha tendido a asociar la violencia escolar con la crisis de las instituciones tradicionales de socialización. 
Dicha crisis se reflejaría en la generación de sujetos anómicos, que exaltan el relativismo moral y desdeñan el respeto por los lazos intergeneracionales que anteriormente cohesionaban a la sociedad. Esto impulsaría a la juventud a realizar acciones de riesgo que naturalizan las soluciones de fuerza, produciendo una "cultura de la violencia" transversal e indiscriminada (Silva, 2006).

Otra matriz estructural-culturalista, ahora de cuño fenomenológico, ha interpretado la violencia escolar como la respuesta de los estudiantes ante la deriva existencial y ontológica característica de la "era del vacío" (Lipovetsky, 1986). De acuerdo con este tipo de análisis:

El horizonte de la violencia tiene raíces antropológicas y existenciales que desbordan los hechos registrados de violencia física, al detectar un quiebre y una crisis más amplia, pero no por ello menos concreta, como es la falta de sentido existencial (...). En otras palabras, la violencia escolar vehicula un sentimiento que está fuertemente ligado con un sentimiento de pérdida y desarraigo existencial (Flores, 2004, pp. 2-3).

De esta manera, el desarraigo y el "riesgo" característicos de la sociedad posmoderna serían fuerzas inabordables para las escuelas, particularmente en el contexto nacional (Sandoval, 2006). Los embates a la antigua "pax escolar" representarían el intento de construir un lugar en el mundo, resignificar las relaciones sociales y restablecer las jerarquías en el contexto de una experiencia escolar "vaciada" (Capriles, 2008; Flores y Zerón, 2007; Vásquez, 2002).

\subsection{Crítica a las interpretaciones estructurales}

La principal limitación del análisis estructural es que al proporcionar una explicación general de la violencia escolar terminan por "descargar" el contenido específico de la misma. Esta subsunción de lo específico (violencia escolar) en lo general (violencia social) supone que, en última instancia, la problemática en estudio no contiene ninguna particularidad, pues, sería simplemente la manifestación 
refleja de procesos sociales mayores ${ }^{4}$. En el fondo, el escenario escolar funcionaría exclusivamente como correa de transmisión de las tensiones de la sociedad.

Un segundo escollo que presenta la interpretación estructuralista es la presunción de que la violencia es unívoca en su origen y uniforme en su despliegue. Los autores adscritos a esta perspectiva tienden a problematizar la noción de violencia como una única forma de manifestación social y escolar, omitiendo la posibilidad de la existencia de múltiples tipos de violencia con lógicas particulares y manifestaciones relativamente autónomas ${ }^{5}$. En algunos casos se habla de distintos factores operantes en la violencia social y/o escolar e incluso se puede inferir la preexistencia de una concepción que distingue distintas violencias, mas, ninguno delimita rigurosamente la existencia de múltiples tipos de violencia, ni expone la especificidad de aquellas que se entrecruzan en el espacio escolar ${ }^{6}$. En este contexto, y como se verá posteriormente, la aceptación de la existencia de una pluralidad de violencias con diversas lógicas de producción requiere de un abordaje particularizado. Es por ello que se hace indispensable elaborar un análisis que se afinque en la especificidad de cada una de estas para, desde allí, recomponer el sentido general de la violencia escolar.

\subsection{Interpretaciones específicas y estudio de casos}

Las interpretaciones que asumen una perspectiva específica tienden a indagar en el fenómeno a partir del estudio de caso, regularmente provienen de alguna de las ramas de la sicología y su análisis se basa en resultados obtenidos a través de material empírico. Sin embargo,

$4 \quad$ Esta es, probablemente, la razón por la cual los propulsores de esta perspectiva han tenido una prolífica producción bibliográfica en la modalidad del ensayo. Mas, esta situación contrasta con el menor volumen de investigaciones empíricas directas que respalden sus postulados en torno a la violencia escolar.

5 Es interesante apreciar que muchos de los que utilizan un marco conceptual y una perspectiva de análisis posmoderna que tiende a relevar la fragmentación, la diversidad y el descentramiento de los fenómenos y las experiencias sociales, insistan en una noción homogeneizante de la violencia, sin advertir la pluralidad de sus lógicas.

6 No se trata de tipos de violencia de acuerdo al "soporte" o las manifestaciones concretas (física, sicológica, simbólica, etc.), sino de la lógica interpretativa que subyace para explicar su origen, actores y direccionalidad. 
en relación con su matriz teórico-conceptual adolecen de limitaciones similares a las revisadas anteriormente, pues, generalmente y más allá de la pretendida "asepsia" teorizante que muchas pretenden establecer, se erigen sobre los mismos paradigmas de base o inciden en un reduccionismo, ahora de signo opuesto, al de las interpretaciones estructurales.

Dentro de estos estudios se pueden distinguir dos directrices de investigación. Por una parte se encuentran los estudios cuantitativos orientados a identificar la prevalencia y la correlación entre los factores determinantes de la violencia escolar. Este tipo de estudios son los más abundantes a nivel internacional y los pioneros en indagar el fenómeno en Chile (Contador, 2001; López y Filsecker, 2001). Estas investigaciones evitan establecer una teorización que engarce los factores correlacionados de la violencia escolar con los procesos que escapan al ámbito educativo. En consecuencia, subyace a este tipo de estudios una adscripción tácita al precepto de la "escuela opaca", presuponiendo que todo lo que ocurre en el escenario educativo estaría determinado por la acción exclusiva de los actores que allí interactúan. En estos casos cobra sentido la sentencia de Teresa Matus:

El asimilar el fenómeno de la violencia escolar a una cuestión de carácter, o a la distribución de golpes (el que los da, el que los recibe) de cada uno de los individuos constituye un velo social, que opaca la cadena de montaje sistémico en que ella se teje (Matus, 2006, p. 33).

Esta crítica ha sido profundizada por otros investigadores nacionales que sostienen que "concebir la violencia escolar solo desde una estructura de acción y reacciones o de conductas y comportamientos individuales, nos hace olvidar el contexto social de emergencia en el cual el fenómeno aparece en las instituciones escolares" (Flores y Retamal, 2011, p. 334) ${ }^{7}$.

Mientras, otros estudios destacan que "al naturalizar las causas de los fenómenos educativos no deseados, se atribuye a características individuales la responsabilidad de las conductas no deseadas por la escuela, "psicologizando" y reduciendo así el análisis del fenómeno educativo" (López et al., 2011, p. 20). 
Un segundo grupo de investigaciones, de carácter cualitativo, ha optado por desentrañar las interpretaciones que construyen los actores escolares sobre la violencia escolar (Berger, Potocnjak y Tomicic, 2011; Calderón, Muñoz J., Muñoz Y., y Lobos, 2010; Flores, 2009; García y Madriaza, 2006; Gómez y Silva, 2011; Guerra et al., 2011; López et al., 2011; Madriaza, 2006; Salgado, 2009; Saavedra, Villalta y Muñoz, 2007; Villalta, Saavedra y Muñoz, 2007)². La indagación centrada en el sentido de la violencia según la subjetividad de los actores escolares ha proporcionado una mayor complejidad en la comprensión de esta problemática. Sin embargo, en la generalidad de estos análisis, el trabajo del investigador se reduce a la recolección y posterior sistematización de información. En el fondo, se incide en la simple descripción de los distintos sentidos de la violencia otorgado por los actores escolares, sin relacionarlos con las prácticas específicas, ni las condiciones materiales y sociales que permiten su expresión. O, cuando esta operación se lleva a cabo, la explicación estructural emerge como horizonte explicativo.

\section{Las figuras de la violencia escolar}

Una de las principales limitaciones que presentan las matrices reseñadas es que tienden a asumir, en su generalidad, que la violencia escolar representa un tipo de manifestación único e indivisible. Por ello, se habla de violencia escolar en singular y tácitamente se asocia la terminología con una de sus expresiones particulares: el bullying. Bajo este registro, por tanto, la violencia escolar se expresaría exclusivamente mediante las agresiones producidas entre los propios estudiantes. El matonaje u hostigamiento, pues, agotaría el espectro general de la violencia escolar.

Por el contrario y a la luz de las nuevas perspectivas esgrimidas por quienes se ocupan de su estudio, sostenemos que la violencia escolar se estructura a través de múltiples manifestaciones que, a su vez, responden a lógicas causales y explicativas disímiles (Dubet,

A pesar de esta separación entre estudios cualitativos y cuantitativos es necesario explicitar que existen investigaciones que han aplicado una metodología mixta para desentrañar el fenómeno de la violencia escolar en Chile (Flores y Zerón, 2007; Valdivieso, 2009). 
1998). En este sentido, no existiría una sola violencia escolar, sino que múltiples violencias caracterizadas por la movilidad de los actores que participan de ella, así como por la diversidad de causalidades que explican su emergencia y reproducción (Bringiotti, Krynveniuk y Lassi, 2007; Míguez, 2008; Sánchez, 2007)9. Dicha pluralidad se expresaría en distintas figuras de la violencia escolar ${ }^{10}$.

La delimitación de estas figuras permitirá clarificar el panorama de la violencia escolar a partir de la reconstrucción de las múltiples manifestaciones que la estructuran. Al respecto, distinguimos tres figuras principales: la violencia de la escuela, la violencia entre pares y la violencia contra la escuela. A continuación se presenta un panorama general de estas figuras, reseñando el tratamiento que de ellas se ha realizado en la investigación nacional ${ }^{11}$.

\subsection{La violencia de la escuela}

Esta se soporta en la teoría de la reproducción postulada inicialmente en las décadas de 1960 y 1970 (Bourdieu y Passeron, 1995). Esta es, por tanto, el primer tipo de violencia escolar que emergió como objeto de estudio específico. La direccionalidad de esta lógica va desde la institución misma y se dirige hacia los estudiantes. Existen investigadores que, sin negar necesariamente la presencia de la violencia simbólica, sostienen que esta es una fórmula muy genérica para comprender las modalidades concretas mediante las cuales los alumnos son violentados cotidianamente. En este contexto, señalan

9 Consecuentemente con este tipo de acercamiento, se ha producido una proliferación de "tipologías" para delimitar las violencias presentes en el espacio escolar. Mientras algunos autores, siguiendo a la sociología francesa y bajo un criterio de direccionalidad, distinguen entre la "violencia de la escuela", la "violencia hacia la escuela" y la "violencia en la escuela" (Di Leo, 2008) otros, que apuntan a identificar el origen desde el cual esta proviene, prefieren hacer la distinción entre la "violencia en la escuela", la violencia "externa a la escuela" y la "violencia antiescolar" (Petrus, 2001) o entre "violencia escolar", "violencia a-escolar" y "violencia anti-escolar" (Zerón, 2006).

10 De acuerdo con Flores: "La idea de 'figura' tiene la ventaja de situar la problemática sobre márgenes distintos a los hechos brutos de la experiencia y las explicaciones causales, para penetrar en una perspectiva hermenéutica y fenomenológica del sentido y 'comprensión' de un fenómeno que se manifiesta de muchas maneras (...). La tarea consiste, entonces, en mostrar y detectar las dinámicas fundamentales y los ejes centrales, de los movimientos internos e invisibles del fenómeno en cuestión" (Flores, 2004, p. 4).

11 Se debe advertir que, en la mayoría de los casos, la adscripción de las investigaciones a alguna de las figuras propuestas es realizada por el autor y no por los mismos autores citados. 
que la violencia de la escuela se ejerce, en la práctica, al exponer a los alumnos al infamante "juicio escolar", aquel que designa la posición que ocupa el sujeto en la escala de éxitos y fracasos académicos (Dubet, 1998; Dubet y Martuccelli, 1998) ${ }^{12}$.

En el escenario educativo chileno, existen estudios que analizan las prácticas cotidianas en la institución escolar, concluyendo que de estas dimanan diversos tipos de violencia (Rojas, 2013) así como operaciones de estigmatización hacia el estudiantado (López et al., 2011). A su vez, Llaña ha explorado la problemática de la conflictividad cotidiana presente en las escuelas nacionales. Si bien su objeto de estudio es la convivencia escolar, el análisis abarca integralmente las condiciones de producción de dicha conflictividad. En este sentido, los estudios de Llaña demuestran que el espacio educativo está cruzado por relaciones de poder que se manifiestan en una normatividad y una práctica pedagógica caracterizadas por el autoritarismo. Por ello, los estudiantes chilenos se muestran violentados por una institución que, bajo una discursividad democratizante, esconde profundos quiebres en torno a la experiencia escolar. Esta situación fomenta un soterrado conflicto que tiende a manifestarse a través de diversas transgresiones que pueden desembocar en expresiones de violencia directa (Llaña, 2010; 2011; Llaña y Escudero, 1999). El carácter coercitivo de la acción institucional también ha sido resaltado en otros estudios acerca de la realidad educativa chilena (Arancibia, 2008), incluyendo el análisis de las normativas institucionales (Carrasco et al., 2014) y la arquitectura escolar como escenario de disciplinamiento (Marambio y Guzmán, 2009).

Los estudios expuestos anteriormente demuestran que la institución escolar produce un tipo de violencia específico caracterizado por orientarse hacia los propios estudiantes. En Chile, esta figura se expresa fundamentalmente a través de un trato vejatorio que degrada la dignidad de los estudiantes y desconoce

12 Las propuestas desarrolladas por la pedagogía crítica y el estudio del currículum oculto también pueden ser inscritos en la perspectiva de la "violencia de la escuela" pues suponen una coacción (cultural, ideológica y/o económica, de género, etaria, etc.) sobre el estudiantado o una parte de este (Giroux, 2001; 2004; Pérez, 2009; Willis, 1977). 
su particularidad sociocultural (López et al., 2011; Neut, 2013; Rojas, 2013).

\subsection{La violencia entre pares}

Esta es la figura que se ha estudiado con mayor extensión y profundidad a nivel internacional y local. La principal característica de la violencia entre pares es que se ejerce entre estudiantes. La manifestación por excelencia de este tipo de violencia escolar es el denominado bullying, hostigamiento o matonaje.

En Chile, el interés por esta figura se ha tornado prácticamente hegemónico. Para su estudio se han utilizado diversas metodologías y analizado particularmente a cada uno de los actores escolares. De este modo, existen estudios que se orientan a investigar la prevalencia (Injuv, 2017) y las interpretaciones que se dan a esta problemática dentro del estamento estudiantil en general (Berger et al., 2011; Calderón et al., 2010; Flores y Zerón, 2007; Gómez y Silva, 2011; Guerra et al., 2011; Tijmes y Fundación Paz Ciudadana, 2012; Saavedra et al., 2007; Valdivieso, 2009). Otros focalizan su atención en los estudiantes que directamente han ejercido la violencia hacia sus pares (García y Madriaza, 2006; Madriaza, 2006; Salgado, 2009). Finalmente, se encuentran aquellos que han inquirido en la interpretación que los profesores realizan en torno al fenómeno (Saavedra et al., 2007) ${ }^{13}$. A su vez, esta figura se ha estudiado a partir de criterios clasificatorios como tipo de dependencia de los colegios (Saavedra et al., 2007) y nivel socioeconómico de los estudiantes (García y Madriaza, 2005; Zerón, 2006).

La sistematicidad de estos estudios ha permitido generar un panorama global respecto de la violencia entre pares presente en Chile. En término generales, se puede afirmar que esta se manifiesta de manera más extendida entre los estudiantes de básica que entre los de educación media, que los varones presentan mayores índices de violencia y victimización respecto del que muestran las mujeres (solo en la violencia de tipo sicológica las mujeres se acercan a los

13 También se han realizado trabajos que incluyen conjuntamente las representaciones de estudiantes y profesores sobre el fenómeno (González, 2010; Neut, 2013). 
índices de los varones), que esta se presenta de manera transversal entre los distintos niveles socioeconómicos (a pesar de que los índices de victimización, clima escolar y, fundamentalmente, percepción de violencia sí presentan diferencias significativas) y que mientras en los estratos bajos se manifiesta de manera más directa una violencia de tipo física en los sectores socioeconómicamente altos predomina aquella de carácter sicológica y verbal.

Como puede apreciarse, la violencia entre pares ha sido, de acuerdo con la cantidad, profundidad y especificación de las investigaciones, la figura de la violencia escolar que ha concitado la mayor atención entre los especialistas chilenos.

\subsection{Violencia contra la escuela}

Esta figura, como constructo teórico específico, ha sido definida precursoramente por Dubet quien sostiene que "son violencia 'antiescuela', las destrucciones de material, los insultos y las agresiones contra los docentes, provocada por los alumnos y, a veces, por su familia y sus amigos" (Dubet, 1998, p. 33). Zerón reafirma la definición de Dubet, reforzando la idea de que esta es "una reacción del alumno contra la autoridad pedagógica” (Zerón, 2004, p. 159). Finalmente, Di Leo señala que la violencia contra la escuela "es aquella que está dirigida hacia los agentes y la infraestructura escolares y, en general, son formas de contestación o reflejo frente a las violencias impuestas por la institución" (Di Leo, 2008, p. 22). La violencia contra o antiescuela, en síntesis, representa aquellos eventos en que la autoridad pedagógica es agredida por algún individuo del estamento estudiantil o alguien "asociado" a él.

En el caso chileno son pocos los estudios que han indagado particularmente en esta figura. El primero corresponde a una tesis de doctorado que verificó la presencia de este tipo de violencia entre escolares de sectores socioeconómicos bajos que asistían a instituciones municipales de Santiago (Zerón, 2006). Posteriormente, un grupo de investigadores ha indagado en la victimización presente en docentes de escuela municipales de la región de Valparaíso (Morales et al., 2014), su relación con las concepciones de la infancia 
(Carrasco et al., 2016) y la normativa reglamentaria desarrollada en escuelas con altos índices de violencia hacia los profesores (Carrasco et al., 2014).

A su vez, el Ministerio del Interior en conjunto con el Ministerio de Educación (a través de otras instituciones a cargo del trabajo de campo: Universidad Alberto Hurtado y Adimark GFK) realizó en tres oportunidades una encuesta bienal sobre violencia en el ámbito escolar. En la última de ellas, efectuada el año 2009, un 11,8\% de los profesores y un 11,3\% de los asistentes de la educación señaló haber sido víctima de violencia (Ministerio del Interior y Mineduc, 2005; 2009). Tras descontinuar la encuesta, el año 2014 se publicó una nueva versión de la misma, constatando un aumento estadísticamente significativo de la violencia anti-escuela. En esta última oportunidad, un $13 \%$ de los docentes encuestados declaró haber sufrido alguna agresión en su establecimiento educativo (Ministerio del Interior, 2014).

Como se aprecia, la violencia antiescuela es la lógica más ignorada por la investigación nacional, a pesar de los estudios exploratorios que reconocen su presencia en las escuelas chilenas. Esta constatación da cuenta de una de las deudas pendientes de la investigación en torno al conocimiento integral de las violencias escolares presentes en el escenario educativo nacional.

\section{Conclusiones}

La violencia escolar es una problemática que interpela tanto a los "especialistas" como al ciudadano común. Sin embargo, y a pesar del rechazo unánime que producen sus expresiones, el tratamiento académico de la misma se ha llevado a cabo incluyendo una buena dosis de confusión. Por un lado, se ha trabajado el tema sin deslindar las matrices teórico-conceptuales que subyacen en las diversas interpretaciones en torno al fenómeno. Por otro, se ha tendido a generar un acercamiento unívoco a esta problemática. En la práctica, la violencia escolar se reduciría a los casos de bullying o violencia entre pares, invisibilizando otras manifestaciones o figuras presentes en las escuelas chilenas. Estas constataciones se transforman en desafíos 
para la comprensión integral de la violencia escolar en Chile, los que operan en diversos niveles o dimensiones.

\section{a. Desafíos para los abordajes teóricos}

A grandes rasgos, la violencia escolar ha sido trabajada como una consecuencia de las tensiones estructurales de la sociedad (matriz estructural), o bien, como el resultado de factores asociados a los sujetos y sus interacciones en el espacio escolar (interpretaciones específicas $)^{14}$. En ambos registros se tiende a producir un reduccionismo interpretativo: uno que vacía el contenido de la violencia que se da en el escenario educativo achacando sus causas a fenómenos que la exceden y sobre los que no tiene incidencia (la violencia social), y otro que, desconociendo los procesos mayores que inciden en la experiencia escolar, descarga todo el potencial explicativo en las características y factores individuales que estimulan comportamientos y relaciones agresivas o violentas.

Al respecto, resulta innegable que la institución escolar está permeada por distintos influjos provenientes de la sociedad. La escuela no es una "isla blindada" de los procesos sociales. En este sentido, se torna necesario comprender la realidad educativa dentro de un proceso de formación multifactorial y pluridimensional. Esta no representa una "caja de resonancia" caracterizada por la recepción pasiva de los procesos sociales, ni se configura de manera completamente autónoma a partir de las dinámicas que se despliegan exclusivamente en su interior. Más bien constituye el resultado de una relación compleja de interdependencia y de mutuas influencias con su entorno.

Asumiendo lo señalado anteriormente, un abordaje adecuado de la problemática en estudio requiere delimitar de la manera más rigurosa posible la relación existente entre la violencia exógena (social, estructural, sistémica, etc.) y su impacto en la generación de acciones o relaciones de violencia al interior de la escuela. En este sentido, se torna necesario reconocer, diferenciar y calibrar el

14 Flores y Retamal engloban a este tipo de interpretaciones bajo la denominación de "paradigma victimizatorio" (Flores y Retamal, 2011). 
efecto de los factores extraescolares (desigualdad, capital cultural, violencia social, etc.) de los propiamente escolares (cultura escolar, interacciones educativas, clima escolar, normativas institucionales, etc.) en la producción específica de cada una de figuras de la violencia. En concreto, y para ilustrar este punto, se puede señalar que la incidencia de los factores externos no es igual en la violencia de la escuela que en la violencia entre pares. En este sentido, la ponderación de los factores no debe ser aplicada de manera uniforme para analizar las distintas figuras de la violencia escolar, sino que requiere de un abordaje particular para cada caso ${ }^{15}$.

Junto con este ejercicio de delimitación de la relación entre la violencia social o exógena y la violencia escolar, se torna indispensable la explicitación de los marcos conceptuales y la figura de la violencia con la que trabaja cada investigación. Esto, debido a que dichas figuras tienen causalidades distintas y, en consecuencia, son relativamente autónomas entre sí. Es por ello que, a nivel teórico, se requiere un análisis de las diversas figuras de la violencia aplicando insumos interpretativos particularizados. Esta es la razón por la cual el rendimiento explicativo de las teorías enunciadas en este trabajo tiene una directa relación con el tipo de violencia que se pretende comprender. Así, por ejemplo, la utilización de un paradigma estructural probablemente posee mayor potencial explicativo para comprender la violencia de la escuela (violencia simbólica, disciplinaria, normativa u otra), sin embargo, pierde eficacia para interpretar la violencia contra la escuela y entre pares.

El reconocimiento de diversas figuras de la violencia y la doble delimitación reseñada (relación violencia exógena/violencia escolar y registro teórico particular/figura de la violencia específica) constituyen un marco general que permite clarificar las matrices conceptuales y que puede servir para expandir el conocimiento en torno a la violencia escolar en Chile.

15 Cuestión importante no solo desde la perspectiva teórica, sino también para la identificación de aquellos aspectos específicos de la escolaridad que estimulan la violencia y que, al emerger dentro de la propia realidad educativa, son susceptibles de ser trabajados y modificados de manera directa. 


\section{b. Desafíos para la investigación}

El interés mayoritario por la investigación en torno al bullying ha permitido generar una comprensión significativa respecto de este tipo de violencia. Sin embargo, una de las consecuencias de esta situación de preeminencia ha sido la estructuración de un conocimiento asimétrico en torno a las diversas figuras de la violencia escolar. En este sentido, y como primer paso para "subsanar" dicha asimetría, se torna indispensable reconocer la existencia de una multiplicidad de violencias que responden a lógicas disímiles, que tienen distinto origen, que involucran -a manera de sujeto u objeto de la violencia- a todos los actores escolares y que se manifiestan de forma simultánea en el escenario educativo.

Esta constatación impele a asumir como desafíos para la investigación futura no solo la profundización del conocimiento en torno a la violencia entre pares, sino también el desentrañamiento de las causas y sentidos de la violencia que la escuela ejerce contra los estudiantes y aquella que estos últimos ejecutan contra la institución y sus autoridades, conocimiento que se torna más apremiante en la medida en que los estudios exploratorios constatan la presencia efectiva de estas otras violencias en las escuelas chilenas

Es, precisamente, la reconstrucción pormenorizada de cada una de estas figuras la que permitirá una comprensión integral de la violencia escolar presente en el escenario educativo nacional.

\section{c. Desafíos para la política pública}

En Chile las políticas públicas en educación han enfatizado progresivamente el tema de la convivencia escolar como un factor relevante para el desarrollo de la comunidad educativa y la formación de los estudiantes. Es así como el año 2002 se publica la primera Politica Nacional de Convivencia Escolar. Este esfuerzo ha sido complementado posteriormente con la promulgación de la Ley $\mathrm{N}^{\circ}$ 20.536 sobre Violencia Escolar ${ }^{16}$, la incorporación de la convivencia

16 A pesar de la intención declarada de fomentar la sana convivencia escolar, esta ley ha sido analizada críticamente por especialistas, tanto en sus principios rectores como en sus disposiciones específicas (Carrasco, López y Estay, 2012; Magendzo, Toledo y Gutiérrez, 2012). 
escolar como un indicador de la calidad educativa y la creación de una nueva Política Nacional de Convivencia el año 2015.

Si bien estos nuevos instrumentos enfatizan el carácter del estudiante como un sujeto de derechos (Mineduc, 2015) y reconocen la importancia de toda la comunidad educativa para la consolidación de una sana convivencia escolar, en la práctica la política pública ha incidido en la misma operación homogeneizante revisada anteriormente en el ámbito de la investigación, reduciendo el fenómeno a la violencia entre pares.

En efecto, si a nivel declarativo se resalta la responsabilidad de todos los actores educativos en el fortalecimiento de la convivencia y la prevención de la violencia escolar, a nivel fáctico los instrumentos aplicados para conocer la violencia escolar se focalizan exclusivamente en la agresión entre pares y el bullying. Resulta indicativo al respecto lo ocurrido con la encuesta bienal sobre violencia en el ámbito escolar. A través de esta, aplicada en tres oportunidades entre los años 2005 y 2009, y luego de manera descontinuada el año 2014, se demostró no solo la presencia sino también el significativo aumento estadístico de las agresiones hacia los docentes en Chile. Sin embargo, y a pesar de esta constatación, dicha variable fue descartada en instrumentos posteriores. En la actualidad, se aplica una encuesta nacional sobre agresión, prevención y acoso escolar a estudiantes que rinden el Simce, medición que solo abarca la violencia entre estudiantes (Mineduc, 2012). A su vez, el último informe acerca de esta materia emitido por un organismo público solo incluye la violencia entre pares (Injuv, 2017).

Lo mismo ocurre con la Ley sobre Violencia Escolar. En esta se define a la misma a través de la noción de acoso, entendido como "toda acción u omisión constitutiva de agresión u hostigamiento reiterado, realizada fuera o dentro del establecimiento educacional por estudiantes que, en forma individual o colectiva, atenten en contra de otro estudiante" (Ley No 20.536). De esta manera, tanto el ordenamiento jurídico como los instrumentos de medición han reducido el problema de la violencia escolar a las agresiones entre estudiantes, invisibilizando las expresiones de violencia "de" y "contra" la escuela. 
En este sentido, se torna urgente que en las políticas públicas no solo se reconozcan las múltiples figuras de la violencia escolar, sino también se diseñen y apliquen instrumentos para conocer su grado de prevalencia a nivel nacional, conocimiento que representa el punto de partida para desarrollar políticas específicas en torno cada uno de los tipos de violencia y, tal como se establece a nivel declarativo, satisfacer de manera integral las demandas por mejorar la convivencia escolar en el escenario educativo chileno.

\section{Referencias}

Arancibia, L. (2008). Relaciones de poder y desigualdad en la escuela secundaria. El caso de Chile de transición y los desafios desde el punto de vista de la democratización. (Tesis para optar al grado de Doctor en Sociología). Universidad Católica de Lovaina, Bélgica.

Ávila, M. (2004). Violencia estructural y resistencia: dos caras de la misma moneda. Revista Electrónica Interuniversitaria de Formación del Profesorado, 5(2), 1-5. Recuperado de: https://dialnet.unirioja.es/ servlet/articulo?codigo $=1034985$.

Berger, C., Potocnjak, M., y Tomicic, T. (2011). Una aproximación relacional a la violencia escolar entre pares en adolescentes chilenos: perspectiva adolescente de los factores intervinientes. Revista Psykhe, 20(2), 39-52. https://doi.org/10.4067/s0718-22282011000200004

Berger, C. y Lisboa, C. (Ed.) (2009). Violencia escolar. Estudios y posibilidades de intervención en Latinoamérica. Santiago de Chile: Editorial Universitaria.

Bourdieu, P. y Passeron, J. (1995). La reproducción. Elementos para una teoría del sistema de enseñanza. Barcelona: Editorial Laia.

Briongiotti, I., Krynveniuk, M., y Lassi, S. (2007). Las múltiples violencias de la 'violencia' en la escuela. Hacia un abordaje integrativo del problema. En G. Averbuj y L. Bozzalla (Comp.), Violencia y escuela. Propuestas para comprender y actuar (pp. 25-48). Buenos Aires: Aique.

Calderón, R., Muñoz, J., Muñoz, Y., y Lobos, K. (2010). Estudio sobre los significados que poseen los estudiantes de sexto y séptimo año básico de tres establecimientos educacionales de la comuna de Valparaíso con respecto a la violencia escolar entre pares. Recuperado de: http://www. academia.edu/1106966/Titulo_Del_Trabajo_ESTUDIO_SOBRE_ LOS_SIGNIFICADOS_QUE_POSEEN_LOS_ESTUDIANTES_DE_ SEXTO_Y_SEPTIMO_ANO_BASICO_DE_TRES_ 
Capriles, C. (2008). Modernidad, educación y violencia: rasgos distintivos de la escuela contemporánea. Ide@a CONCYTEG, 3(36), 164-182. Recuperado de: http://www.concyteg.gob.mx/ideasConcyteg/ Archivos/36122008_MODERNIDAD_EDUCACION_VIOLENCIA.pdf

Carrasco, C., Verdejo, T., Asun, D., Álvarez, J., Bustos, C., Ortiz, S., ... Valdivia, N. (2016). Concepciones de infancia en una escuela con altos índices de violencia escolar de estudiantes a profesores. Revista Latinoamericana de Ciencias Sociales, Niñez y Juventud, 14(2), 11451159. https://doi.org/10.11600/1692715x.14218210814

Carrasco, C., Ortiz, S., Baltar, M., Álvarez, J., Bustos, C., Asun, D., y Verdejo, T. (2014). Violencia de reglamento: análisis de los reglamentos de convivencia escolar en dos escuelas con alto nivel de violencia reportada de estudiantes a profesores de la Región de Valparaíso, Chile. Revista de Estudios Cotidianos, 2(2), 117-137.

Carrasco, C., López, V., y Estay, C. (2012). Análisis crítico de la Ley de Violencia Escolar de Chile. Psicoperspectivas. Individuo y Sociedad, 11(2), 31-55. https://doi.org/10.5027/psicoperspectivas-voll1-issue2-fulltext-228

Cofré, E. (2013). Imaginario social del conflicto en la escuela: alcances de la violencia escolar y el bullying. Némesis: Malestar y Conflicto Social, 86-95. Recuperado de: http://www.academia.edu/4075335/ Imaginario_social_del_conflicto_en_la_escuela_alcances_en_la_ violencia_escolar_y_el_bullying

Contador, M. (2001). Percepción de violencia escolar en estudiantes de enseñanza media. Revista Psykhe, 10(1), 69-80.

Di Leo, P. (2008). Violencias y escuelas: despliegue del problema. En A. L. Kornblit (Coord.), Violencia escolar y climas sociales (pp. 17-41). Buenos Aires: Editorial Biblos.

Dubet, F. (1998). Las figuras de la violencia en la escuela. Revista Francesa de Pedagogía, 132, 35-45.

Dubet, F. y Martuccelli, D. (1998). En la escuela. Sociología de la experiencia escolar. Buenos Aires: Losada.

Flores, L. y Retamal, J. (2011). Clima escolar y gestión compleja del conocimiento: desafíos para la investigación educativa y la política pública en violencia escolar. MAGIS, Revista Internacional de Investigación en Educación, 4(8) 319-338. Recuperado de: http://www. redalyc.org/articulo.oa?id=281021722003

Flores, L. (2009). Interpretaciones fenomenológicas sobre el sentido de la violencia escolar. En Christian Berger \& Carolina Lisboa (eds.). 
Violencia escolar. Estudios y posibilidades de intervención en Latinoamérica (pp. 211-230). Santiago de Chile: Editorial Universitaria

Flores, L. (2004). Horizonte de comprensión de la violencia escolar como fenómeno antropológico. Hacia una recuperación de la subjetividad educativa. Revista Investigaciones en Educación, 4, 67-75.

Flores, L. y Zerón, A. (2007). Paradojas de la violencia escolar en Chile desde la subjetividad fenomenológica de los actores. Boletín de Investigación Educacional, 22, 14-28.

García, M. y Madriaza, P. (2006). Estudio cualitativo de los determinantes de la violencia escolar en Chile. Revista Estudios de Psicología, 11(3), 247-253.

García, M. y Madriaza, P. (2005). Sentido y propósito de la violencia escolar. Análisis estructural del discurso de estudiantes agresivos del nivel socioeconómico alto. Revista de Psicología, 14(1), 41-59. Recuperado de: http://www.revistapsicologia.uchile.cl/index.php/RDP/article/ viewFile/17333/18074

Giroux, H. (1983). Teorías de la reproducción y la resistencia en la nueva sociología de la educación: un análisis crítico. Cuadernos Políticos, 44, 36-65. Recuperado de: http://www.cuadernospoliticos.unam.mx/ cuadernos/contenido/CP.44/cp.44.6.\%20HenryAGiroux.pdf

Giroux, H. (2001). Cultura, política y práctica educativa. Barcelona: Graó.

Giruox, H. (2004). Teoría y resistencia en educación. México DF: Siglo XXI.

Gómez, D. y Silva, C. (2011). Significados de los adolescentes sobre el conflicto, la violencia escolar y estrategias de resolución de conflictos dentro del marco de la convivencia escolar. Revista Observatorio de la Juventud, Injuv, 30, 11-24. Recuperado de: http://www.injuv.gob.cl/ portal/wp-content/files_mf/revistaobservatorio3075.pdf

González, C. (2010). Percepciones sobre violencia en el ámbito escolar y gestión directiva: una aproximación a partir de la fase cuantitativa de un estudio en la Región de Valparaíso. Psicoperspectivas. Individuo y Sociedad, 9(2), 105-135.

https://doi.org/10.5027/psicoperspectivas-vol9-issue2-fulltext-122

Guerra, C., Álvarez, D., Dobarro, A., Núñez, J., Castro, L., y Vargas, J. (2011). Violencia escolar en estudiantes de educación secundaria de Valparaíso (Chile): comparación con una muestra española. Revista Iberoamericana de Psicología y Salud, 2(1), 75-98. Recuperado de: http://www.redalyc. org/articulo.oa?id=245116403004

Instituto Nacional de la Juventud, Injuv. (2017). Sondeo $N^{\circ}$ 5: Bullying en establecimientos educacionales. Recuperado de: http://www.injuv. 
gob.cl/portal/wp-content/uploads//2017/05/resultados-sondeo-05bullying.pdf

Ley No 20.536 Sobre Violencia Escolar de Ministerio de Educación. Diario Oficial de la República de Chile, 17 de septiembre de 2011.

Lipovetsky, G. (1986). La era del vacío: ensayos sobre el individualismo contemporáneo. Barcelona: Anagrama.

López, V., Carrasco, C., Morales, M., Ayala, A., López, J., y Karmy, M. (2011). Individualizando la violencia escolar: análisis de prácticas discursivas en una escuela municipal de la Región de Valparaíso. Revista Psykhe, 20(2), 7-23. https://doi.org/10.4067/s0718-22282011000200002

López, M. y Filsecker, M. (2001). Manifestaciones de violencia y agresividad en la escuela: estudio comparativo en tres realidades geográficas distintas. Santiago: ICET.

Llaña, M. (2010). Convivencia escolar: disímiles construcciones simbólicas en oposición. Revista Enfoques Educacionales, 11(1), 55-64. Recuperado de: http://www.facso.uchile.cl/publicaciones/enfoques/11/enfoques_ n11_2009_2010.pdf

Llaña, M. (2011). La convivencia en los espacios escolares. Una incursión hacia su invisibilidad. Santiago de Chile: Facultad de Ciencias Sociales, Universidad de Chile.

Llaña, M. y Escudero, E. (1999). Establecimientos educacionales en conflicto: grupos de resistencia en su interior. Revista Enfoques Educacionales, 2(2), 155-166.

Magendzo, A., Toledo, M., y Gutiérrez, V. (2012). Descripción y análisis de la Ley sobre Violencia Escolar ( $\mathrm{N}^{\circ}$ 20.536): dos paradigmas antagónicos. Estudios Pedagógicos, 39(1), 377-391.

https://doi.org/10.4067/s0718-07052013000100022

Madriaza, P. (2006). Sentido social de la violencia escolar. Transformaciones culturales en el discurso marginal de los que están sujetos de la violencia (Tesis inédita para optar al grado de Magíster en Antropología y Desarrollo). Universidad de Chile, Santiago.

Madriaza, P., Varela, T., y Nespolo, R. (2011). Reflexiones en torno a la violencia escolar en América Latina, ¿En qué estamos? Boletín $\mathrm{N}^{\circ}$ 12, Instituto de Asuntos Públicos, Universidad de Chile. Recuperado de: http://www.pazciudadana.cl/wp-content/ uploads/2013/07/2011-12-30_Reflexiones-en-torno-a-la-violenciaescolar-en-Am\%C3\%83\%C2\%A9rica-Latina-\%C3\%82\%C2\%BFenqu\%C3\%83\%C2\%A9-estamos.pdf 
Marambio, J. y Guzmán, S. (2009). La construcción de la escuela como espacio carcelario: una representación social de los jóvenes relativa a la (sobre) vivencia (en) de su espacio escolar. En J. Redondo (Ed.), Juventud y enseñanza media en Chile del Bicentenario, antecedentes de la revolución pingüina (pp. 59-91). Santiago de Chile: OPECH.

Matus, T. (2006). Naturalizaciones violentas. Análisis de algunas paradojas existentes en las investigaciones psicológicas y sociales en fenómenos complejos como la violencia escolar. Revista Trabajo Social, 73, 31-44.

Míguez, D. (2008). ¿Violencia en las escuelas? La cuestión en perspectiva. En D. Míguez (Comp.), Violencias y conflictos en las escuelas (Introducción). Buenos Aires: Paidós.

Ministerio de Educación de Chile, Mineduc. (2015). Política Nacional de Convivencia Escolar, 2015-2018. Recuperado de: http://www. mineduc.cl/wp-content/uploads/sites/19/2015/12/politica-noviembredefinitiva.pdf

Ministerio de Educación de Chile, Mineduc. (2012). Resultados nacionales agresión, prevención y acoso escolar Simce 2012. Recuperado de: https://s3.amazonaws.com/archivos.agenciaeducacion.cl/ informesresultados2012/Resultados+nacionales+agresion,+prevenci on+y+acoso+escolar_+SIMCE+2012.pdf

Ministerio del Interior (2014). Encuesta Nacional de Violencia en el Ámbito Escolar (ENVAE). Recuperado de: http://www.seguridadpublica.gov. cl/media/2016/11/Presentaci\%C3\%B3n-ENVAE.pdf

Ministerio del Interior y Mineduc. (2005). Primera Encuesta de Violencia en el Ámbito Escolar. Recuperado de: http://www.seguridadpublica.gov.cl/ filesapp/pres_ppt.pdf

Ministerio del Interior y Mineduc. (2009). Tercera Encuesta de Violencia en el Ámbito Escolar. Recuperado de: http://www.seguridadpublica.gov.cl/ filesapp/presentacion_violencia_escolar_2009_web.pdf

Morales, M., Álvarez, J., Ayala, A., Ascorra, P., Bilbao, M., Carrasco, ... y Villalobos, B. (2014). Violencia escolar a profesores: conductas de victimización reportadas por docentes de enseñanza básica. Revista de Estudios Cotidianos, 2, 1-116. Recuperado de: http://www. estudioscotidianos.com/index.php/estudioscotidianosl/article/view/50

Neut, P. (2013). Las lógicas de la violencia contra la escuela. Aproximación desde las formas de configuración de la autoridad pedagógica actual (Tesis inédita para optar al grado de Magíster en Educación). Universidad de Chile, Santiago. 
Noel, G. (2009). La conflictividad cotidiana en el escenario escolar. Buenos Aires: UNSAM EDITA.

Palomero, J. y Fernández, M. (2001). La violencia escolar, un punto de vista global. Revista Electrónica Interuniversitaria de Formación del Profesorado, 41, 19-38.

Pérez, A. (2009). Las funciones sociales de la escuela: de la reproducción a la reconstrucción crítica del conocimiento y la experiencia. Laboratorio de Políticas Públicas, 6, 1-46. Recuperado de: http://biblioteca.clacso. edu.ar/Argentina/lpp/20100324022908/9.pdf

Petrus, R. (2001). Cultura de la violencia y educación secundaria. Revista Española de Educación Comparada, 7, 23-49. Recuperado de: http:// revistas.uned.es/index.php/REEC/article/view/7323/6991

Rojas, J. (2013). Clima escolar y tipología docente: la violencia escolar en las prácticas educativas. Cuadernos de Investigación Educativa, 4(19), 87104. https://doi.org/10.18861/cied.2013.4.19.28

Sánchez, M. (2007). La mirada de docentes y alumnos sobre la violencia en la escuela. En G. Averbuj y L. Bozzalla (Comp.), Violencia y escuela. Propuestas para comprender y actuar (pp. 169-482). Buenos Aires: Aique.

Salgado, F. (2009). Representaciones sociales acerca de la violencia escolar. Revista Iberoamericana sobre Calidad, Eficacia y Cambio en Educación, 7(3), 138-152. Recuperado de: https://revistas.uam.es/index.php/reice/ article/view/5405

Saavedra, E., Villalta, M., y Muñoz, M. (2007). Violencia escolar: la mirada de los docentes. Revista Limite, 15, 39-60. Recuperado de: http://www. redalyc.org/articulo.oa?id=83601503

Sandoval, M. (2006). La violencia escolar desde la teoría del riesgo y el cambio cultural. Santiago de Chile: Investigaciones CEJU, Centro de Estudios en Juventud, UCSH.

Silva, A. (2006). La cultura de la violencia: la transgresión y el miedo de los adolescentes. Revista Fermentum, 47, 664-674. Recuperado de: http:// www.redalyc.org/html/705/70504705/

Tijmes, C. y Fundación Paz Ciudadana (2012). Violencia y clima escolar en establecimientos educacionales en contextos de alta vulnerabilidad social de Santiago de Chile. Revista Psykhe, 21(2), 105-117. https://doi.org/10.7764/psykhe.21.2.548

Valdivieso, P. (2009). Violencia escolar y relaciones intergrupales. Sus prácticas y significados en las escuelas secundarias públicas de la comuna de Peñalolén 
en Santiago de Chile (Tesis inédita para optar al grado de Doctor en Psicología). Universidad de Granada, España.

Vásquez, J. (2002). Crisis y paradojas de la modernidad al hilo del discurso escolar. Política y Sociedad, 39(3), 707-721. Recuperado de: https:// dialnet.unirioja.es/servlet/articulo?codigo $=623611$

Villalta, M., Saavedra, E., y Muñoz, M. (2007). "Pasado a llevar". La violencia en la educación media municipalizada. Revista Estudios Pedagógicos, 33(1), 45-62. http://dx.doi.org/10.4067/S0718-07052007000100003

Willis, P. (1977). Aprendiendo a trabajar. Madrid: Akal.

Zerón, A. (2006). Sentido de la violencia escolar en Chile. Un estudio de sociología comprensiva (Tesis inédita para optar al grado de Doctor en Ciencias de la Educación). Pontificia Universidad Católica de Chile, Santiago de Chile.

Zerón, A. (2004). Violencia escolar y violencia anti-escuela. Aportes teóricos para una aproximación sociológica. Boletín de Investigación Educacional, 19(2), 43-61.

Recibido: 15/08/2016

Aceptado: 15/05/2017 